\title{
Ngugi wa Thiong'o et la décolonisation par la langue
}

Xavier Garnier

\section{(2) OpenEdition}

\section{Journals}

Édition électronique

URL : http://journals.openedition.org/actesbranly/488

DOI : $10.4000 /$ actesbranly.488

ISSN : 2105-2735

Éditeur

Musée du quai Branly Jacques Chirac

Référence électronique

Xavier Garnier, «Ngugi wa Thiong'o et la décolonisation par la langue », Les actes de colloques du musée du quai Branly Jacques Chirac [En ligne], 3 | 2011, mis en ligne le 26 avril 2011, consulté le 08 septembre 2020. URL : http://journals.openedition.org/actesbranly/488 ; DOI : https://doi.org/ $10.4000 /$ actesbranly.488

Ce document a été généré automatiquement le 8 septembre 2020

(c) Tous droits réservés 


\title{
Ngugi wa Thiong'o et la décolonisation par la langue
}

\author{
Xavier Garnier
}

1 Je vais donc parler de l'écrivain kenyan Ngugi wa Thiong'o, pas dans la totalité de son œuvre, mais pour me consacrer à la partie de sa vie où il a décidé de cesser de faire sa création littéraire en langue anglaise et de ne la faire plus qu'en Kikuyu.

2 Ngugi wa Thiong'o est un écrivain kényan né en 1938, à Limuru au Kenya. Il s'agit d'une figure d'écrivain engagé qui n'a jamais cessé de mêler les préoccupations esthétiques et politiques. Il passe l'année 1978 en prison et prend alors la décision de ne plus écrire son œuvre littéraire qu'en Gikuyu. Il engage un combat pour l'utilisation des langues africaines dans l'expression littéraire. On a souvent assimilé cette décision à l'adoption d'une position "afrocentriste » : il y a ici un malentendu à dissiper... Il me semble que le sens de ce combat peut être mis en perspective du point de vue du sujet qui nous préoccupe aujourd'hui : la question des « littératures noires» ne se pose pas vraiment pour Ngugi, je voudrais montrer pourquoi elle est secondaire pour lui et comment le passage au Gikuyu va paradoxalement l'orienter du côté d'une « littérature-monde ».

Les littératures en langues africaines avant 1978 : je ne reviens pas sur la question des manuscrits, notamment ajami, pour ne m'intéresser qu'à la littérature imprimée. Je retiens deux temps principaux: la littérature sous influence missionnaire et la littérature sous influence coloniale... Les missions apportent les imprimeries, elles impriment des traductions de la Bible, de textes édifiants (cas célèbre du Pilgrim's Progress de John Bunyan). Pour ce faire elles font un gros travail de préparation des langues: standardisation, choix pour la graphie. Le lieu où sont installées les presses est déterminant pour le choix du dialecte appelé à devenir standard... Il s'agit d'une littérature pragmatique, à vocation locale. C'est dans cette dynamique que vont apparaître de nombreux écrivains au début $\mathrm{du} \mathrm{xx}^{\mathrm{e}}$ siècle, qui prennent un tournant nationaliste. Ce sont souvent des catéchistes, qui ont assisté les missionnaires dans le développement d'une littérature nationaliste kikuyu... comme Thomas Mofolo pour le Sotho, Vilakazi pour le Zoulou, Modalités du contrôle colonial sur cette littérature : La création d'une presse coloniale en langues africaines est liée à la volonté de contrôler, 
voire d'infléchir, ce qui s'écrit dans les langues africaines (cf. exemple de Léon Mamboleo en Afrique de l'Est). On installe un peu partout, du moins en Afrique anglophone, des literature bureau, qui organisent des concours littéraires avec prix, langues par langues. Le cas le plus global est le Prix Margaret Wrong: promotion institutionnelle d'une littérature en langues africaines, encadrée (avec cahier des charges, etc.). Cette littérature est invitée à répondre à des attentes nettement culturalistes (et fort peu politiques).

Ouverture du débat au moment des Indépendances. Dès le congrès de 1956, David Diop aborde la question dans sa «contribution au débat sur la poésie nationale. En 1957, Albert Memmi écrit dans son Portrait du colonisé :

«L'écrivain colonisé, péniblement arrivé à l'utilisation des langues européennes celle des colonisateurs, ne l'oublions pas -, ne peut que s'en servir pour réclamer en faveur de la sienne. Ce n'est là ni incohérence ni revendication pure ou aveugle ressentiment, mais une nécessité. Ne le ferait-il pas, que tout son peuple finirait par s'y mettre. Il s'agit d'une dynamique objective qu'il alimente certes, mais qui se nourrit seule également et continuerait de toute façon sans lui. Ce faisant, il contribue à liquider son drame d'homme, il confirme, il accentue son drame d'écrivain. Pour concilier son destin avec lui-même, il pourrait s'essayer à écrire dans sa langue maternelle. Mais on ne refait pas un tel apprentissage dans une vie d'homme. L'écrivain colonisé est condamné à vivre ses divorces jusqu'à sa mort. Le problème ne peut se clore que de deux manières : par tarissement naturel de la littérature colonisée; les prochaines générations, nées dans la liberté, écriront spontanément dans leur langue retrouvée. Sans attendre si loin, une autre possibilité peut tenter l'écrivain : décider d'appartenir totalement à la littérature métropolitaine. Laissons de côté les problèmes éthiques soulevés par une telle attitude. C'est alors le suicide de la littérature colonisée. Dans les deux perspectives, seule l'échéance différant, la littérature colonisée de langue européenne semble condamnée à mourir jeune. » (Gallimard, « Folio », 2002 : 128.)

5 Dans le monde anglophone le débat est ouvert en 1962 à un congrès à l'université de Makerere (Ouganda) par Obi Wali, (article publié dans la revue Transition, $\left.\mathrm{n}^{\circ} 10\right]$ : «The Dead end of African literature » [ « Le cul de sac de la littérature africaine »] dans une communication jugée par Ngugi lui-même comme agressive et méprisante.

Récapitulatif des positions au tournant des années 60: a) En faveur des langues européennes : nécessité d'un dialogue panafricain - possibilité d'africaniser ces langues (Achebe, Okara, Tutuola), b) Contre les langues africaines : langues n'étant pas prêtes pour l'écrit - risque d'enfermement dans des ghettos culturels (cf. politique de l'apartheid et le refus de Marechera d'écrire en shona), c) En faveur des langues africaines : être en contact avec les masses (Obi Wali) ou les peuples (David Diop, Albert Memmi), d) Contre les langues européennes : jouer le jeu de l'assimilation c'est donner raison à la colonisation.

7 La position de Ngugi au début des années 1980 : Ngugi est reconnu comme un écrivain anglophone majeur (4 romans, 2 pièces de théâtre, de nombreux articles...) et fait le choix d'écrire en Gikuyu. Il s'explique dès 1981, dans un article au titre ambigu: «Return to the Roots». Beaucoup de commentateurs y ont vu le signe d'un repli identitaire sur la communauté gikuyu. En 1986, il consacre un livre entier à cette décision: Decolonising the Mind. Simon Gikandi consacre un article très riche à cette conversion de Ngugi $^{1}$ et insiste sur les tensions et les contradictions entre une conception matérialiste - la langue est dépendante de ses conditions de production (Ngugi marxiste) et une conception idéaliste -la langue est un vecteur de l'unité 
spirituelle d'un peuple (Ngugi nationaliste). Le passage à l'écriture en Gikuyu est présenté comme une conversion en 2 étapes, racontée dans Decolonising the mind (1986).

Il y a deux moments complémentaires dans son récit, qui correspondent à deux étapes initiatiques: d'une part, une expérience de mise en scène d'une pièce de théâtre en Gikuyu dans le centre culturel Kamĩriinthũ à Limuru; expérience collective de fusion avec une population locale, participant activement aux répétitions, et intervenant dans le processus créatif. Ngugi insiste sur la jubilation qu'il éprouve à être dessaisi de son œuvre... d'autre part, l'expérience de la prison : isolement total, l'écrivain se retrouve dans un face à face solitaire avec la langue et les mots. Il fait alors l'expérience de leur opacité, de leur matérialité (à travers les graffitis, les journaux qui servent d'emballage par exemple) et en même temps de leur intensité dès lors que le monde extérieur est suspendu à eux. L'homme est nié, le rapport aux textes est une question de survie... (il n'est plus rien d'autre qu'un écrivain). Ces deux expériences sont à mettre en relation avec une conception « quasi-mystique » de la création littéraire.

9 La prise en compte des écueils : Ngugi identifie clairement les arguments de ceux qui refusent d'avoir recours aux langues africaines. Il y répond implicitement par son positionnement :

1. [argument du didactisme] il ne s'agit pas de faire une littérature pour le peuple (nouvelle version de la littérature missionnaire), mais plutôt par le peuple. Le langage, comme communication, est le témoin de la constitution en acte d'une communauté. Il n'y a pas de communauté sans dynamique créative... Les phénomènes de rumeur sont au principe de l'écriture des trois romans écrits en Gikuyu : Devil on the cross (1980), Matigari (1986) et Wizard of the crow (2004).

2. [argument de l'enfermement ethnique] il ne s'agit pas d'exprimer «l'âme gikuyu », mais de trouver un point de perspective sur le monde: la langue n'est pas le reflet d'une culture comme contenu, mais elle devient culture dès lors qu'elle s'ouvre au monde depuis un lieu. Importance de la notion de local chez Ngugi (Ngugi wa Thiong'o, «The universality of local knowledge », in Moving the centre. The struggle for cultural freedom, London, James Currey, 1993, p. 25-29). Limuru est un lieu, à partir duquel on a une perspective sur le monde, mais qui n'est pas une position de domination. Bouger le centre, signifie multiplier les centres à l'infini, comme autant de points de vue sur le monde. Les problèmes commencent quand on se sert du langage pour se voir soi-même, depuis un point de vue extérieur à soi (qu'il appelle le point de vue de l'aliénation) [argument de l'archaïsme de ces langues]. Il n'y a pas de jugement négatif sur la disposition des langues européennes à se propager. Toutes les langues ont une vocation à l'expansion et ce phénomène n'est pas nécessairement en soi générateur de conflits. « S'il n'y a rien de mal, pour quelque groupe que ce soit, à essayer de promouvoir l'extension de sa langue, il est entièrement mauvais pour un groupe, un peuple, une communauté, une nation, une race, de devenir à tel point fasciné par les langues des autres que l'on commence à voir dans sa propre langue une barrière au progrès et à la modernité (Ngugi wa Thiong'o, «Return to the Roots. Language, culture and politics in Kenya » in Writers in politics. A reengagement with issues of literature \& society, Londres, James Currey, $1997[1981]: 63)^{2}$. Les choses tournent mal dès lors qu'on intériorise un sentiment d'infériorité et qu'on bloque cette dynamique. Cela est valable à l'intérieur des langues ellesmêmes entre les différents dialectes ou patois. On a reproché à Ngugi d'avoir recours au Gikuyu de Limuru : c'est celui dont il a fait «l'expérience ».

3. La notion d'« expérience » au cœur de la démarche de Ngugi : la notion de «langue natale » est importante pour Ngugi, mais moins comme langue transmise par les ancêtres, que comme langue parlée dans l'enfance. On fait dans l'enfance une expérience émotive du monde par le langage, cette expérience est au cœur de la culture. C'est pourquoi la culture ne saurait être objectivée, mais toujours expérimentée (positions très proches de celle de 
Okot p'Bitek à la même époque), le problème de la littérature africaine en langues européennes est de constituer un corpus culturel « objectif » à partir duquel on risque de discriminer les gens en "plus ou moins cultivés ». Ngugi plaide pour une généralisation de l'activité de traduction: il faut traduire les chefs d'œuvres mondiaux en gikuyu, pour permettre à tous d'en faire «l'expérience» (sur la traduction intra-africaine, lire Decolonising the Mind, 1986 : 84-85). Cette notion « d'expérience » permet d'expliquer sa prise de distance avec la fiction réaliste à l'occasion du changement de langue : représenter le monde suivant les conventions du roman réaliste est une modalité d'expérience du monde qui a partie liée avec l'expérience des langues occidentales, voir sur ce point l'article de Christine Loffin, dans Research in African Literature $(26-4,1995)$ sur la progressive disparition du descriptif dans les romans en anglais comme signe avant-coureur de la "conversion » linguistique...

10 Ngugi propose à la littérature africaine d'oublier un peu l'Afrique pour s'intéresser au monde depuis un lieu concret, qui corresponde à l'expérience de chacun. Cela apparaît clairement dans la dédicace de Matigari: «Lecteur/auditeur : puisses-tu placer cette action dans le lieu de ton choix ${ }^{3}$ !»

11 Un point important pour finir : Il s'agit de se défaire d'une obsession de l'Afrique et de l'africanité, par le recours à une expérience concrète du monde depuis un lieu concret. Si l'on veut parler d'un afrocentrisme de Ngugi, alors il faut comprendre qu'il s'agit d'abord d'un "souci du monde ", et qu'il est en ce sens beaucoup plus proche qu'on pourrait le penser des intentions affichées des écrivains de la « littérature-monde ».

\section{ANNEXES}

\section{Débat}

\section{Alain Ricard}

Je voudrais poser une question à Romuald Fonkoua sur la place qu'accorde justement la revue Présence Africaine aux langues africaines?

\section{Romuald Fonkoua}

Depuis 1956, le débat sur la place accordée aux langues nationales fait régulièrement surface. Ce débat est posé en termes sociolinguistiques, plutôt qu'en termes littéraires. La question est d'autant plus compliquée qu'elle se pose à un moment où les sociétés africaines elles-mêmes, n'ont pas encore une religion sur cette question-là. Je ne suis pas certain d'ailleurs que la plupart des pays ont aujourd'hui une religion sur cette question-là. Le débat est donc posé assez régulièrement. Des linguistes se demandant comment on peut inventer, construire une langue nationale dans tel ou tel pays. Il est vrai que la revue n'est pas allée du côté des littératures en langues africaines.

\section{Question du public}


Bonjour, je m'appelle Jean Thebault et j'étais extrêmement intéressé par cette dernière intervention. Cela m'a rappelé des débats qui eurent lieu entre les deux guerres à propos des langues régionales.

\section{Xavier Garnier}

Il y a certainement des volontés politiques d'aménagement des langues... Il existe un risque qui est celui de ne pas faire intervenir les écrivains dans ce processus de standardisation de la langue. Il est important que les décideurs soient sensibilisés à l'importance que peuvent avoir les écrivains dans ce processus-là.

\section{Florence Paravy}

C'est une question pour Xavier Garnier. Tu as traité des auteurs principalement anglophones, et ce que tu as dit sur le rôle des missionnaires, etc., est aussi vrai ailleurs?

\section{Xavier Garnier}

Oui, il y a une différence mais je n'en connais pas la raison. L'implantation des missionnaires est souvent antérieure à la conférence de Berlin. C'est beaucoup plus dans les zones anglophones que les missions ont développées vraiment cette littérature en langues africaines. C'est un peu un mystère.

\section{Lilyan Kestelloot}

En effet, il y a de grandes différences en Afrique. Ce n'est pas du tout la même situation. La politique coloniale en Afrique francophone a été radicalement différente. Non seulement les missionnaires, etc. ont peut-être été très actifs, mais la politique coloniale anglaise a également permis l'enseignement des langues nationales, en tout cas dans la plupart des pays comme le Nigéria, etc. Tandis que jamais, durant toute la colonisation, la France n'a autorisé que les langues nationales entrent à l'école. Chez nous, les enfants sont alphabétisés directement en français et ça les coupe, évidemment. Cela a porté un préjudice plus important que dans les pays anglophones où les langues nationales n'ont pas disparu comme dans les pays francophones. Il suffit de lire le livre de Ricard pour s'en rendre compte. Aujourd'hui tout le problème c'est la récupération de ces langues. Le gros problème chez nous, c'est que les éditeurs refusent en bloc de publier en langues nationales. Moi, j'en sais quelque chose, car je travaille surtout sur les problèmes de transcription des littératures orales. Il ne s'agit donc pas de mes livres, mais des livres des autres. Je vois déjà la difficulté en Afrique d'arriver à faire publier... Et en France c'est pire.

\section{NOTES}

1. S. Gikandi, « Ngugi's conversion: Writing and the Politics of Language ", Research in African Literature 23(1) (numéro spécial « The question of language ») : 131-144.

2. "While there is nothing wrong with any group to promote the spread of their language, there is something entirely wrong in any group, people, community, nation, race, becoming so mesmerized by other's people's languages that they begin to look at their own languages as barriers to progress and modernity. »

3. «And it has no fixed space./ Here or there... / This or that village... / This or that region. / Reader/listener : may you place the action in the space of your choice!» (Matigari, p. IX) 


\section{AUTEUR}

\section{XAVIER GARNIER}

Il enseigne les littératures francophones à l'Université Paris 3. Ses travaux portent sur la littérature en Afrique et sur la théorie du roman. Il s'intéresse notamment à la question des littératures en langues africaines et a publié en 2006, aux éditions Karthala, un ouvrage sur le roman swahili. 